\title{
PULSE-WAVE ELECTROCOMA
}

\author{
BY
}

\section{E. B. STRAUSS, J. GOULD, and ANGUS MacPHAIL}

\author{
From the Department of Psychological Medicine, St. Bartholomew's Hospital, London
}

There have been many articles dealing with the clinical value of electrocoma, as we prefer to call "electronarcosis", in the course of the last half dozen years. Some writers make considerable claims for the technique in the treatment of schizophrenia and other mental disorders, but a few have been less favourably impressed. This contribution to the literature is intended to be technical and methodological rather than clinical.

Although we have been treating patients by electrocoma for the last four years, we do not feel entitled yet to express a firm opinion as to the value of the treatment because (1) the total number of patients treated at St. Bartholomew's Hospital to date, although " respectable", is insufficient for statistical purposes; (2) all our patients have been treated under out-patient conditions, and none have received more than one treatment a week; (3) there is no means of judging whether a series of electrically induced convulsions given at the rate of one a week would have produced the same clinical results as electrocoma. The only method of arriving at an opinion on this point would have been to have treated a number of patients by electroplexy and another clinically comparable control group by electrocoma and to have assessed the results accordingly. We were not in a position to do this. We must be content, therefore, to record a clinical impression to the effect that certain patients, notably early cases of paranoid schizophrenia and the obsessional form of psychosis, have derived benefit from the treatment. We have noted improvement in a number of patients exhibiting phobic anxiety states with obsessional features. And the results are sufficiently encouraging to warrant continued investigation.

It is some years now since Frostig, Van Harreveld, Reznick, Tyler, and Wiersma (1944) applied direct current, interrupted direct current, and alternating current to the brain, and later, together with Tietz, Thompson, Van Harreveld, and Wiersma (1945) evolved the technique which came to be known as electronarcosis.
Their first experiments were conducted with dogs and made use of an interrupted direct current produced by a thyratron-excited square-wave generator. They found that they could induce coma with a pulse of about 140 microseconds' duration with a repetition-frequency of anything from 120 to 1,000 per second. If alternating current is used, frequencies of from 30 to 8,000 cycles per second are effective, but the coma is more disturbed with frequencies that exceed 500 .

Some six years ago Tietz made use of an apparatus which passed an alternating current of mains frequency through the brain in such a way that the current level could be pre-set and maintained in spite of the rapid changes in the patient's impedance. Tietz's original circuit included a power amplifier with an appropriate negative feed-back, but in our original version of this apparatus we used a high-voltage source of alternating current and a large series ballast resistor. Our object was to prevent changes in the patient's resistance materially affecting the total impedance of the circuit, thus enabling the current to be maintained at the desired level. Others have used magnetic stabilizers to attain the same end (MacPhail, 1949).

Although all these apparatus deliver 50-60 cycle alternating current to the patient and can produce and maintain coma for minutes at least, the risks involved impressed us as being unnecessarily high : respiration is apt to be seriously embarrassed, with consequent stridor and cyanosis; there is a dramatic rise of blood-pressure, often as much as 30 or 40 points ; cardiac irregularities, including fibrillation, are temporarily produced ; a condition amounting to tetany requires to be maintained if a satisfactory coma is to be kept up ; it is almost impossible to produce or maintain coma without a preliminary convulsion. This is not a disadvantage in itself, but it makes it extremely difficult to decide whether the clinical results of the treatment are to be attributed to coma or to electroplexy (E.C.T.).

We had one death from what appeared to be central heart failure during coma induced by a 


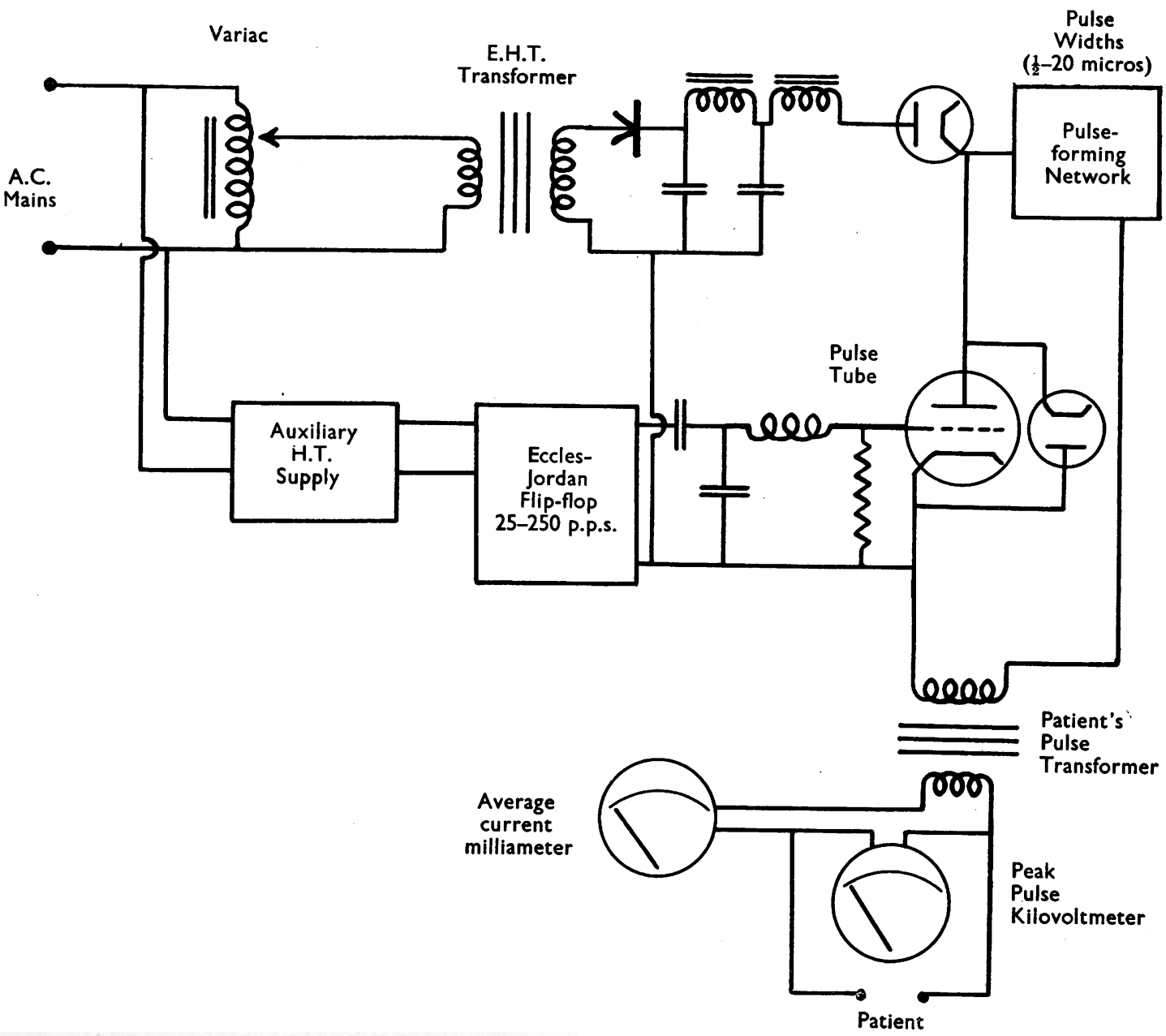

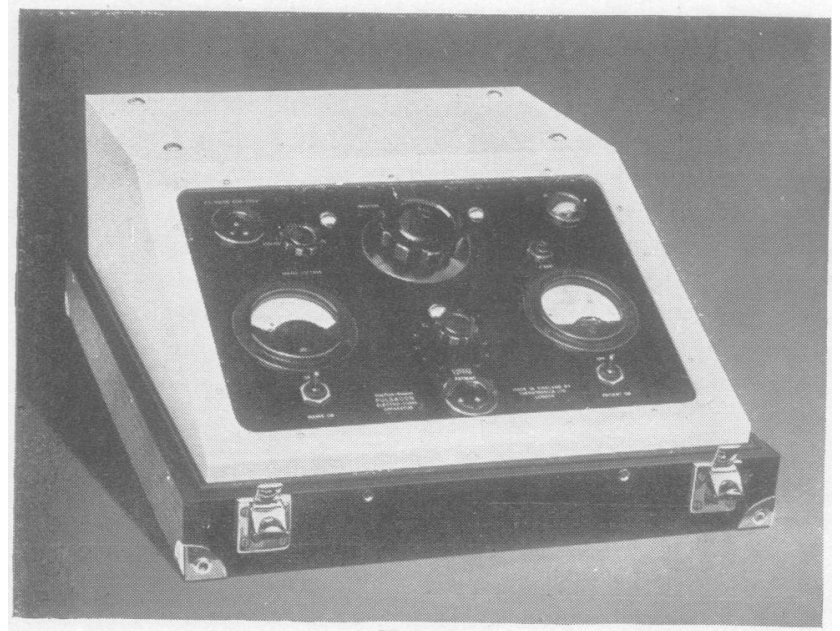

FIG. 2

FIG. 1

50-cycle current. The patient, a young leucotomized woman, had had three previous inductions of electrocoma and appeared to be benefiting from the treatment. All efforts at resuscitation by the anaesthetists in attendance proved unavailing. We therefore decided to experiment with a wide range of electrical wave-forms in an attempt to induce coma with a large margin of safety for the patient and as little anxiety as possible to the operator: we wished to reduce the physiological side-effects to a minimum compared with those which seemed inseparable from the use of raw alternating current.

We finally decided on trains of pulses of from $\frac{1}{2}-20$ microseconds with repetition-frequencies of 50-200 per second as being the most desirable. 
The peak pulse voltages applied to the patient range up to 3 kilovolts. This range represents previously unheard-of peak voltages and currents, and induces an entirely new set of electrical reactions and demonstrates that the human brain can with complete safety support high-voltage currents of very short pulse duration continuously. The watt-second rate is the determining factor.

The electrical circuit used in this work is illustrated in Fig. 1. It consists of a high-voltage transformer fed from the A.C. mains through a "variac". A direct high tension supply is made available by rectifying the output of this transformer, and smoothing by means of condensers and choke.

An auxiliary high tension supply is fed to a modified Eccles-Jordan flip-flop oscillator. This drives the main pulse-tube which generates high voltage pulses to the pulse-forming network, finally delivering to the patient who acts as the load. Pulse voltage is variable from 0 to $2 \cdot 2$ kilovolts ; the pulse width from 0.5 to 20 microseconds. The peak pulse kilovoltage is shown on an electrostatic voltmeter. The average current to the patient during treatment is indicated by a moving coil milliammeter. The energy factor is selected by a rotary switch which also regulates the pulse width in microseconds. The outward appearance of the apparatus with its carrying cover removed, showing the control panel, can be seen in Fig. 2. This instrument, the "pulsacon", is manufactured by Messrs. Theratronics, Ltd., 6, Argyle Walk, London, W.C.1.

\section{Technique}

Preparation of Patient.-The patient should have had no food for the previous four hours. He is given $1 \frac{1}{2}$ gr. " epanutin" by mouth about an hour before treatment and a subcutaneous injection of atropine sulphate, gr. 1/100, about half an hour later. The same sort of couch and hard pillow for hyperextending the back as are used in electroplexy are employed in electrocoma, and, as in electroplexy, a solid rubber gag is placed between the patient's teeth.

A tight-fitting rubber bathing cap (we have found this to be the most suitable form of head-gear) is fitted over the head in such a way that the anterior edge reaches the line of the eyebrows. The patient is now lightly anaesthetized with an intravenous barbiturate. We find that $0.5 \mathrm{~g}$. thiopentone usually suffices. The electrodes are now placed in position under the bathing cap. Each consists of a foil of stainless steel, $1 \frac{5}{8}$ in. by $1 \frac{3}{8}$ in., sandwiched in $\frac{1}{4}$ in.thick slabs of cellulose sponge. The sponge and the metal electrode are retained in position by being sewn into a little envelope of lint. Each electrode

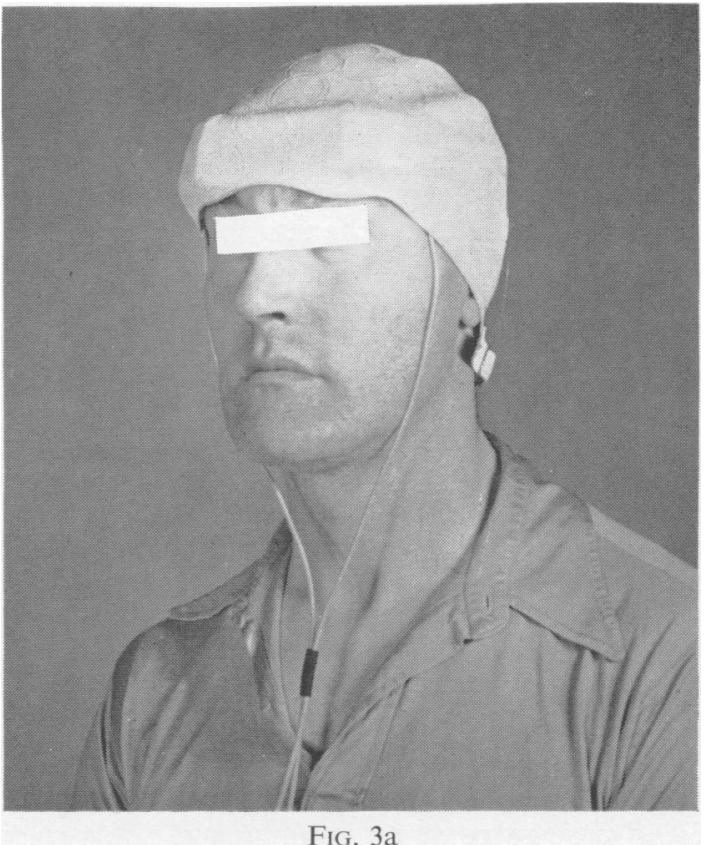

unit is soaked in lukewarm 10 to $20 \%$ saline. The correct position of the electrodes is such that the lower lengthwise edge is $\frac{1}{2}$ in. above the mid-eyebrow level, with the proximal anterior edge of the electrode half-way between the mid-pupillary line and the outer canthus of the eye (Fig. 3).

It is important that the electrodes should be accurately placed, as too low a position is likely to cause excessive laryngeal spasm, and a too medial position produces too light a coma. Counterpressure is applied to the shoulders and hips, although the muscular contractions resulting from

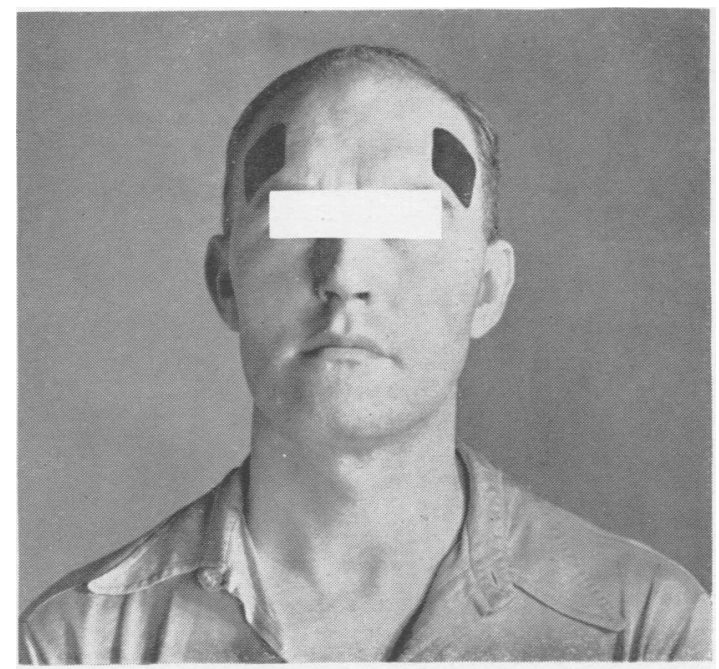

FIG. $3 b$ 
the initial stimulation are less tetanic and still less tonic than in electroplexy. In this connexion, it is worth noting that out of a total of some 500 treatments only one (doubtful) lamellar crush was observed, and this occurred in the case of a very muscular young man who had not been anaesthetized with thiopentone.

Electrical Dosage Values.-We usually induce coma with the following electrical values : $1.5 \mathrm{Pkv}$; pulse repetition-frequency, 105 per second ; pulsewidth, measured in microseconds, 10 ; milliamperage, 9. The current is passed for 30 seconds, the patient having been previously oxygenated by the anaesthetist. There is an immediate contraction of skeletal muscle which is sub-maximal and tetanic in character, but cyanosis is not observed. The contracted muscles make it impossible to feel the pulse at the wrist or at the neck, but the femoral pulse is not affected. There is an immediate rapid rise in the pulse rate. The apex beat is frequently obscured, and the heart sounds are often inaudible on auscultation.

The current is reduced after the first half-minute by diminishing the pulse-width and lowering the kilovoltage, which rises automatically as the pulse width is reduced, so as to keep the kilovoltage for a while at its original level of $1.5 \mathrm{Pkv}$. This may be reduced later to $1.3 \mathrm{Pkv}$. The pulse-width is reduced from its original level of 10 microseconds to 3.3 microseconds, or sometimes, especially in the case of muscular young men, to 5 microseconds. The milliamperage falls proportionately to the current and the kilovoltage and will now be about between 3 and 5 m.a. Respiration is spontaneously re-established within 10 to 20 seconds, frequently with some stertor or stridor owing to laryngeal spasm. The respiratory rate may be as high as 40 per minute at this stage although it is more commonly under 35 . The pulse rate may be as high as $\mathbf{1 6 0}$ for a short time, but usually falls rapidly. Dropped beats and extra-systoles often occur at this stage of treatment.

Diminution of pulse-width is followed by a reduction of muscle tone. With a pulse-width of 3.3 microsecs. an observer can with ease effect passive movements of the limbs. Further reduction of the pulse-width, say to 1 microsec., is accompanied by considerable generalized hypotonia and relaxation.

The pulse rate is usually high throughout coma ; this would appear to be a central tachycardia, as, after the first $\mathbf{3 0}$ seconds of induction, there is no cardiac or respiratory strain or embarrassment. When the current is finally switched off, the pulse rapidly returns to normal.
From this stage of the treatment onwards the pulse-width is slowly reduced to the lowest value consistent with the continuation of coma, maintaining the voltage at the $1.5 \mathrm{Pkv}$. level. It will be remembered that the milliamperage varies automatically with the pulse-width.

When the electrically induced coma has been maintained for from one to four minutes, the patient may become slightly restless and begin to phonate. This does not appear to be identical with the hyperkinesis described by Frostig; it is more likely to be a manifestation of subcortical activity. Further reduction of the electrical stimulus in terms of kilovoltage, or preferably pulse width, restores tranquillity and allows coma to proceed smoothly. Oxygen, which is given before and during induction, can usually be discontinued after the first 45 seconds.

The time during which smooth coma may be continued varies from patient to patient. Powerfully built young males as a rule require a greater electrical dosage than others, and even then they frequently come out of coma after four or five minutes, and rarely remain comatose for longer than eight minutes, whatever the stimulus. Slightly built, young female patients are the easiest to keep comatose and the coma may be maintained for up to a quarter of an hour, if desired. It is generally agreed that the optimum length of treatment is seven minutes.

When coma is prolonged neurological signs similar to those associated with decortication may be observed ; for example, the posture is decorticate, the plantar responses are extensor, and the kneeand ankle jerks tend to be increased. These signs disappear quickly and are no longer to be elicited two minutes after the cessation of coma.

The ordinary criteria by which one judges the depth of anaesthesia do not apply in electrocoma ; the respiratory rhythm is different from that associated with anaesthesia produced by other methods, expiration following inspiration without pause, the whole respiratory sequence being firm and forceful. The eyelids remain tightly closed, and lachrymation may occur as the resuit of local radiation of the current to the muscles of the eyelids. If the eyelids are prised open within 30 seconds of the thiopentone injection or at any time during coma, the pupils are seen to be contracted. Termination of coma is soon followed by dilatation of the pupils.

At first, we interpreted the type of phonation as evidence of lightness of anaesthesia or impending termination of coma. We no longer believe this to be the case, as even a slight reduction of the electrical stimuhus abolishes this sort of subcortical 
activity. If the treatment is artificially interrupted at this stage, the patient does not immediately regain full consciousness but falls asleep for up to 20 minutes or more.

The likelihood that this pseudo-purposeful behaviour of the restless kind is subcortical rather than cortical in nature is supported by observations on patients who were given electrocoma without thiopentone anaesthetization. Some of these patients, who were given an electrical stimulus which was insufficient to produce smooth coma and exhibited restless motor patterns, subsequently described subjective experiences of a crude, emotionally over-invested nature which were too bizarre to be properly verbalized. One patient, for example, described great balls of fire and a feeling as though he were approaching the Judgment Seat ; another patient was aware of increasing tension amounting to horror. A third patient became so conditioned that he would fly from the sight of electrical apparatus no matter where it was. On one occasion he saw some electrical appliances being unloaded from a military lorry and was quite unable to restrain himself from making a dash into the road.
These conditioned reactions do not occur with thiopentone.

\section{Summary and Conclusions}

Although we are not yet prepared to assess the clinical value of electrocoma in psychiatric practice, it is, in our opinion, a form of electrocerebral treatment which should continue to be investigated.

We believe that a pulse-wave current allowing of four variables-pulse-width, repetition frequency, peak voltage and milliamperage-provide the best and safest method of inducing and maintaining coma.

An apparatus which fulfils these requirements and its method of employment are described in detail.

\section{REFERENCES}

Frostig, J. P., Van Harreveld, A., Reznick, S., Tyler, D. B., and Wiersma, C. A. G. (1944). Arch. Neurol. Psychiat., Chicago, 51, 232.

Tietz, E. B., Thompson, G. N., Van Harreveld, A., and Wiersma, C. A. G. (1945). Amer. J. Psychiat., 101, 821.

MacPhail, A. (1949). Electronics Forum, No. 14, p. 27.

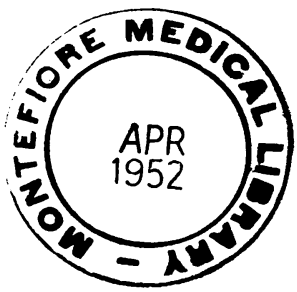

